\title{
Therapy for Multiple Myeloma - Quo Vadis?
}

\author{
Leo Rasche, ${ }^{1}$ Klaus Martin Kortuem ${ }^{1}$ and Hermann Einsele ${ }^{2}$
}

1. Consultant Physician; 2. Director, Department of Internal Medicine II, Hematology and Oncology, University Hospital of Wuerzburg, Wuerzburg, Germany

\begin{abstract}
Two major developments are changing our current understanding of cancer: (1) the in-depth analysis of tumour-harbouring mutations and (2) the generation of highly active T cell-involving immunotherapies. In this editorial, we focus on its impact on myeloma therapy and speculate on future treatment concepts.

\section{Keywords}

Multiple myeloma, next-generation sequencing, actionable mutations, targeted therapy, monoclonal antibodies, CAR T cells, BiTE antibodies

Disclosure: Leo Rasche and Klaus Martin Kortuem have received funding from the Deutsche Forschungsgemeinschaft. Hermann Einsele has received honoria for lectures and participation in advisory boards of Amgen, Celgene, Novartis and Janssen. No funding was received in the publication of this article.

Open Access: This article is published under the Creative Commons Attribution Noncommercial License, which permits any non-commercial use, distribution, adaptation and reproduction provided the original author(s) and source are given appropriate credit. DOl: $\mathrm{http}$ ://doi.org/10.17925/EOH.2015.11.02.107

Received: 2 September 2015 Published Online: 16 November 2015 Citation: European Oncology \& Haematology, 2015;11(2):107-8

Correspondence: Leo Rasche, Department of Hematology and Oncology, University Hospital of Wuerzburg, Oberduerrbacher Str 6, 97080 Wuerzburg, Germany.

E: Rasche_L@ukw.de
\end{abstract}

Multiple myeloma (MM) therapy has undergone dramatic changes over the last decades, including the use of autologous haematopoietic cell transplants and the development of generations of cereblonand proteasome-inhibiting drugs. This progress has translated into a significant improvement in survival for standard risk patients and, even long-term disease control, and maybe a cure, can be achieved at least in a subset of patients. However, two recent developments bear the potential to further revolutionise our current understanding of cancer, and will particularly impact myeloma therapy. The first is the widespread use of next-generation sequencing (NGS) techniques, as well as, second, the development of active immunotherapies, such as monoclonal antibodies, checkpoint inhibitors, bispecific antibodies or chimeric antigen receptor (CAR) T cells.

In 2011, Chapman and colleagues first reported on a NGS-based analysis of 38 newly diagnosed MM patients using whole genome/ whole exome sequencing and found a high degree of heterogeneity among the patients, but also a number of recurrent mutated genes. Among those, most frequently point mutations in KRAS and NRAS had been present in about the half of the cases; in another patient a BRAFV600E mutation was found. ${ }^{1}$ Larger studies including more than 1,000 exomes followed and confirmed mutations in the MAP kinase pathway in around $50 \%$ of the patients. ${ }^{2-5}$ This is of importance since the MAP kinase pathway is potentially druggable/actionable with molecular therapeutics, such as MEK or BRAF inhibitors. Trametinib is an oral, allosteric inhibitor of MEK1/2 blocking the MAP kinase pathway downstream from RAF and that has been tested in patients with activating mutations in the MAPK pathway. Heuck et al. recently reported on 59 heavily pre-treated patients with most of them bearing alterations in the MAP kinase pathway having received trametinib alone $(n=22)$ or in combination with other myeloma drugs upfront or later during the course of treatment. Clinical benefit response rate (overall response rate [ORR] + minor response) was $>55 \%$. Of note, six patients experienced complete resolution of focal lesions in positron emission tomography/computed tomography (PET/CT) scans while being treated with single-agent trametinib; furthermore, high-risk extramedullary cases responded. The authors state that their results are reminiscent of their early investigations into thalidome. ${ }^{6}$ Andrulis et al. reported on a patient with extramedullary disease harbouring a BRAFV600E mutation in which a partial remission could be achieved by treatment with the BRAF inhibitor vemurafinib and several other reports followed and confirmed efficacy of vemurafenib in BRAF600E mutated cases even at late or refractory stages. ${ }^{7-9}$ In a recent analysis of 463 newly diagnosed and homogeneously treated MM patients from the National Cancer Research Institute (NCRI) Myeloma XI trial, Walker and colleagues, for the first time, linked mutations in a number of genes to the individual survival of MM patients. They identified a total number of 309 actionable targets in $53 \%$ of patients and the authors speculate to increase to 440 targets applicable to $62 \%$ of patients in the near future (see Figure 1, green puzzles). Thus, a personalised precision medicine using molecular therapeutics directed to actionable mutations will constitute a new therapeutic concept. ${ }^{4}$

Another field of progress are immunotherapeutic agents in the treatment of $\mathrm{MM}$, in particular the monoclonal antibodies, checkpoint inhibitors and engineered T-cell concepts. The concept of immunotherapy in MM is well established since the use of allogeneic stem cell transplantation and interferon $\alpha$ treatment. However, an equivalent to the efficacy of CD20 antibodies had been missing so far. This gap should already be filled. Just recently, elotuzumab, an anti-SLAMF7 immunoglobulin G (IgG) antibody, showed improved ORR (79 versus $66 \%$ ) and progressionfree survival (PFS) (19.4 versus 14.9 months) in combination with 


\section{Figure 1: Molecular and Immunotherapeutic Targets of Multiple Myeloma}

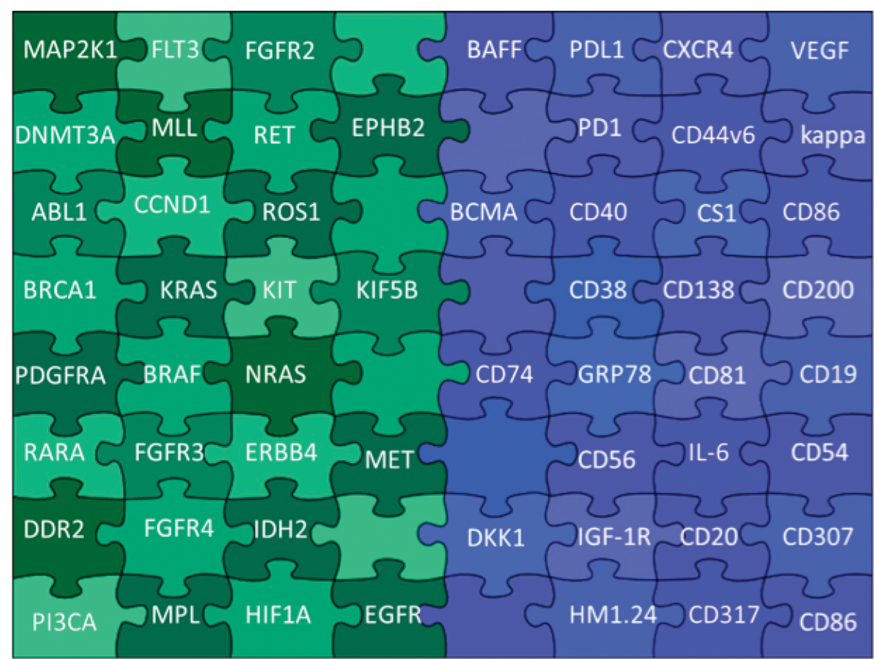

Green puzzles show actionable mutations found in multiple myeloma patients. Blue puzzles represent immunotherapeutic targets which are under investigation in preclinical and clinical trials.

Ienalidomide plus dexamethasone (Len/Dex)-treated patients in a randomised phase III study without adding toxicity to the treatment. ${ }^{10}$ Daratumumab and SAR650984, both IgG1 antibodies targeting CD38, have shown remarkable single-agent activity. ORR of these antibodies varied between 58 and $92 \%$ when used in combination with Len/Dex in early-phase clinical trials, including responses in patients that had relapsed after pomalidomide or carfilzomib treatment. 11,12 $^{1,1}$

Promising data have been additionally obtained from studies evaluating T cell or T-cell targeting therapies. Checkpoint inhibitors, such as nivolumab or pembrolizimumab, showed impressive responses and durable remissions in drug refractory smoking associated lung cancer, illustrating the potency of T-cell-based therapies. Furthermore, tumour-associated antigens can be highly efficiently targeted by CAR T cells or bispecific T-cell-engaging antibodies (BiTES). At present this success is still limited to CD19-positive disease, but a considerable number of new targets are under investigation (see Figure 1, blue puzzles). ${ }^{13,14}$ One example is the B cell maturation antigen (BCMA), which is stably expressed on myeloma cells and is involved in tumour proliferation and progression. Both BCMA directed CAR T cells as well as BiTE antibodies show promising in vitro activity and are currently evaluated in early clinical trials. 15,16 Interestingly, CD19 addressing CAR $\mathrm{T}$ cells may also be used in $\mathrm{MM}$ to eradicate disease propagating precursor cells after a salvage auto transplant. Two heavily pre-treated and relapsed patients underwent such strategy and experienced complete remission. ${ }^{17}$

With regard to checkpoint inhibitors, preliminary studies in MM had been disappointing. Twenty-seven patients had received programmed cell death protein 1 (PD-1) antibody nivolumab in a dose-escalating schedule in a phase I trial and, apart from disease stabilisation in 18 subjects, no objective responses occurred. ${ }^{18}$ However, we believe the concept of PD-1 or programmed death-ligand 1 (PD-L1) interfering therapy is a powerful concept for active immune stimulation in particular in combination with other immunomodulating myeloma drugs.

In summary, a number of novel drug targets are currently investigated and specific compounds including monoclonal antibodies will soon be part of our drug arsenal of anti-MM compounds. Individualised treatment regimens will be performed based on next-generation sequencing of the individual tumours, and customised therapies will be applied. Never before has the future looked brighter for MM patients to reach the aim of long-term disease control or maybe even a cure in a significant subset of MM.
1. Chanman MA, Lawrence MS, Keats IJ, et al., Initial genome sequencing and analysis of multiple myeloma, Nature 2011:471:467-72

2. Bolli N, Avet-Loiseau H, Wedge DC, et al., Heterogeneity of genomic evolution and mutational profiles in multiple myeloma, Nat Commun, 2014;5:2997.

3. Lohr JG, Stojanov P, Carter SL, et al., Widespread genetic heterogeneity in multiple myeloma: implications for targeted therapy, Cancer Cell, 2014;25:91-101.

4. Walker BA, Boyle EM, Wardell CP, et al., Mutational spectrum, copy number changes, and outcome: Results of a sequencing study of patients with newly diagnosed myeloma, J Clin Study of patients with newly diagn 2015 [Epub ahead of print].

5. Lonial S, Yellapantula VD, Liang W, et al., Interim analysis of the Mmrf Commpass Trial: Identification of novel rearrangements potentially associated with disease initiation and progression, Blood, 2014:124(21).

6. Heuck CJ, Jethava Y, Khan R, et al., Inhibiting MEK in MAPK pathway activated myeloma, Leukemia, 2015 [Epub ahead of print].

7. Sharman JP, Chmielecki J, Morosini D, et al., Vemurafenib response in 2 patients with posttransplant refractory BRAF V600E-mutated multiple myeloma, Clin Lymphoma Myeloma
Leuk, 2014:14(5):e161-3.

8. Andrulis M, Lehners N, Capper D, et al., Targeting the BRA V600E mutation in multiple myeloma, Cancer Discov 2013;3:862-9

9. Bohn OL, HSU K, Hyman DM, et al., BRAF V600E mutation and clonal evolution in a patient with relapsed refractory myeloma with plasmablastic differentiation, Clin Lymphoma Myeloma Leuk, 2014;14:e65-8.

10. Lonial S, Dimopoulos M, Palumbo A, et al., Elotuzumab therapy for relapsed or refractory multiple myeloma N Engl J Med, 2015;373:621-31.

1. Martin TG, Baz R, Benson Jr. DM, et al, A Phase ib Dose Escalation Trial of SAR650984 (Anti-CD-38 mAb) in Escalation Trial of SAR650984 (Anti-CD-38 mAb) in Combintion Relapsed/Refractory Muliple Myeloma, Presented at: 56 th ASH Annual Meeting \& Exposition, San Francisco, CA, September 2014

12. Plesner, T., et al., Safety and Efficacy of Daratumumab with Lenalidomide and Dexamethasone in Relapsed or Relapsed, Refractory Multiple Myeloma, Presented at: 56th ASH Annual Meeting \& Exposition, San Francisco, CA, 7 September 2014.

13. Topp MS, Gökbuget N, Stein AS, et al., Safety and activity of blinatumomab for adult patients with relapsed or refractory B-precursor acute lymphoblastic leukaemia: a multicentre, single-arm, phase 2 study, Lancet Oncol, 2015;16:57-66.

14. Lee DW, Kochenderfer JN, Stetler-Stevenson M, et al., $\mathrm{T}$ cells expressing CD19 chimeric antigen receptors for acute lymphoblastic leukaemia in children and young adults: a phase 1 dose-escalation trial, Lancet, 2015 7;385:517-28.

15. Ramadoss NS, Schulman AD, Choi SH, et al., An anti-B cell maturation antigen bispecific antibody for multiple myeloma, J Am Chem Soc, 2015;137:5288-91.

16. Carpenter, R.O., et al., B-cell maturation antigen is a promising target for adoptive T-cell therapy of multiple promising target for adoptive T-cell therapy of
myeloma, Clin Cancer Res, 2013:19:2048-60.

17. Garfall AL,, Safety and efficacy of anti-CD19 chimeric antigen receptor (CAR)-modified autologous T cells (CTLO19) in advanced multiple myeloma, Presented at 2015 ASCO Annua Meeting, Chicago, II, 1 June 2015

18. Lesokhin AM, Ansell SM, Armand P, et al., Preliminary results of a phase I study of nivolumab (BMS-936558) in patients with relapsed or refractory lymphoid malignancies, Presented at: 56th ASH Annual Meeting \& Exposition, San Francisco, CA, 7 September 2014 